\title{
TREATMENT OF REFRACTORY ACUTE ALLOGRAFT REJECTION WITH AEROSOLIZED CYCLOSPORINE IN LUNG TRANSPLANT RECIPIENTS
}

Robert J. Keenan, MD

Aldo Iacono, MD

James H. Dauber, MD

Adriana Zeevi, PhD

Samuel A. Yousem, MD

N. Paul Ohori, MD

Gilbert J. Burckart, DPharm

Akihiko Kawai, MD

Gerald C. Smaldone, MD

Bartley P. Griffith, MD
Lung transplant recipients who have persistent acute cellular rejection are at increased risk for the development of chronic rejection, the leading cause of reduced long-term survival. This study evaluated the use of aerosolized cyclosporine as rescue therapy for unremitting acute rejection. Between June 1993 and March 1996, 18 patients with rejection that failed to resolve after therapy with pulse steroids and antilymphocyte globulin were enrolled in the study. Aerosolized cyclosporine A (300 mg) treatment was initiated for 10 consecutive days followed by a maintenance regimen of 3 days per week. Efficacy was assessed by graft histologic and pulmonary function testing. With the use of linear regression, results in these patients were compared with those in 23 control patients, matched for histologic acute rejection, who had continued to receive conventional rescue therapy. Two patients were unable to tolerate the treatments and were withdrawn from the study. Significant improvement in histologic rejection occurred in 14 of the remaining 16 patients after a mean of 37 days of aerosolized cyclosporine therapy. Measures of forced vital capacity and forced expiratory volume in 1 second (change in percent predicted/100 days plus or minus the standard error) increased over time in the treated patients whereas the condition of control patients declined despite repeated attempts at conventional rescue (forced vital capacity, aerosolized cyclosporine group, $4.6 \pm$ 2.9 vs control group $-8.1 \pm 1.9, p=0.001$; forced expiratory volume in 1 second, aerosolized cyclosporine group, $2.1 \pm 4.4$ vs control group $-9.8 \pm$ 2.6, $p=0.043$ ). Renal and hepatic toxicity during cyclosporine therapy was not observed. The incidence of acute histologic rejection ( $\geq A 2$ ) decreased from $2.49 \pm 0.68$ episodes $/ 100$ days before aerosolized cyclosporine therapy to $0.72 \pm 0.3$ episodes $/ 100$ days $(p<0.05$ ). In summary, aerosolized cyclosporine is a safe and effective therapy for acute rejection that has failed to improve with conventional treatment. (J Thorac Cardiovasc Surg 1997;113:335-41)
From the Departments of Surgery, Medicine, Pathology, and Pharmacy and Therapeutics, University of Pittsburgh, Pittsburgh, Pa., and the Department of Medicine, State University of New York at Stony Brook, Stony Brook, N.Y.

Supported by Food and Drug Administration drug grant RFAFD-OP-90-1.

Read at the Seventy-sixth Annual Meeting of The American Association for Thoracic Surgery, San Diego, Calif., April 28-May 1, 1996.

Received for publication May 1, 1996; revisions requested June 17, 1996; revisions received Sept. 6, 1996; accepted for publication Sept. 11, 1996.

Address for reprints: Robert J. Keenan, MD, Division of Cardiothoracic Surgery, C-702 PUH, University of Pittsburgh Medical Center, Pittsburgh, PA 15213.

Copyright (C) 1997 by Mosby-Year Book, Inc.

$0022-5223 / 97 \$ 5.00+0 \quad \mathbf{1 2 / 6 / 7 8 0 3 5}$
Triple-drug regimens (cyclosporine or tacrolimus, azathioprine, prednisone) are routinely used as the maintenance therapy for lung transplant recipients. Despite the success of these protocols, acute rejection affects more than $75 \%$ of patients. ${ }^{1-5}$ Episodes of acute cellular rejection are usually treated with short-term enhancement of immunosuppression consisting of pulse corticosteroids or cytolytic therapy. These efforts are not always successful and persistent or refractory acute rejection develops in many recipients. $^{1-4}$ If acute rejection is not controlled it invariably results in tissue injury and organ failure. Persistent acute rejection is the predominant risk factor for the subsequent development of chronic rejection or death within the first year after transplantation. 3, 4,6 
Table I. Patient demographics and lung function study results immediately before aerosolized cyclosporine therapy

\begin{tabular}{|c|c|c|c|c|c|c|c|c|c|c|c|}
\hline \multirow[b]{2}{*}{ Patient } & \multirow[b]{2}{*}{ Age (yr) } & \multirow[b]{2}{*}{$\mathrm{Sex}$} & \multirow[b]{2}{*}{ Pre-Tx Dx } & \multirow[b]{2}{*}{ Organs } & \multirow[b]{2}{*}{ Day $R x$} & \multicolumn{2}{|c|}{$F V C$} & \multicolumn{2}{|c|}{$F E V_{1}$} & \multicolumn{2}{|c|}{$F E F_{25-75}$} \\
\hline & & & & & & $L$ & $\% *$ & $L$ & $\% *$ & $L / \mathrm{sec}$ & $\%$ \\
\hline 1 & 31 & $\mathrm{M}$ & $\mathrm{PF}$ & L. single & 91 & 1.25 & 23 & 1.20 & 28 & 2.26 & 51 \\
\hline 2 & 42 & $\mathrm{~F}$ & $\mathrm{PH}$ & R. single & 246 & 3.31 & 98 & 2.62 & 100 & 2.41 & 78 \\
\hline 3 & 51 & $\mathrm{M}$ & COPD & R. single & 515 & 3.85 & 73 & 1.77 & 46 & 0.63 & 17 \\
\hline 4 & 24 & $F$ & $\mathrm{CF}$ & Double & 190 & 2.32 & 61 & 1.86 & 60 & 1.91 & 53 \\
\hline 5 & 26 & $\mathrm{M}$ & $\mathrm{PH}$ & Double & 271 & 3.60 & 62 & 1.90 & 42 & 0.76 & 16 \\
\hline 6 & 53 & $\mathrm{~F}$ & COPD & R. single & 386 & 2.06 & 69 & .85 & 38 & 0.23 & 9 \\
\hline 7 & 26 & $\mathrm{~F}$ & $\mathrm{CF}$ & Double & 339 & 2.57 & 78 & 1.92 & 72 & 1.54 & 47 \\
\hline 8 & 61 & $F$ & COPD & R. single & 568 & 1.67 & 68 & .82 & 46 & 0.19 & 8 \\
\hline 9 & 50 & $\mathrm{M}$ & COPD & L. single & 525 & 2.28 & 50 & 1.35 & 39 & 0.56 & 16 \\
\hline 10 & 52 & $\mathrm{~F}$ & COPD & R. single & 275 & 2.18 & 73 & 1.15 & 51 & 0.44 & 16 \\
\hline 11 & 40 & $\mathrm{M}$ & $\mathrm{CF}$ & Double & 321 & 1.88 & 39 & 0.84 & 23 & 0.31 & 8 \\
\hline 12 & 43 & $\mathrm{M}$ & $\mathrm{CF}$ & Double & 782 & 3.20 & 61 & 3.02 & 76 & 4.17 & 104 \\
\hline 13 & 57 & $\mathrm{M}$ & COPD & R. single & 863 & 2.66 & 60 & 0.8 & 25 & 0.63 & 20 \\
\hline 14 & 55 & $\mathrm{~F}$ & COPD & R. single & 398 & 1.27 & 45 & 0.79 & 38 & 0.45 & 18 \\
\hline 15 & 20 & M & CF & Double & 723 & 2.20 & 64 & 1.38 & 44 & 0.68 & 19 \\
\hline 16 & 28 & $\mathrm{M}$ & $\mathrm{CF}$ & Double & 816 & 3.78 & 88 & 2.95 & 82 & 2.58 & 61 \\
\hline 17 & 59 & $\mathrm{~F}$ & COPD & R. single & 318 & 1.68 & 57 & 1.16 & 53 & 0.65 & 26 \\
\hline 18 & 43 & $F$ & $\mathrm{PH}$ & Double & 767 & 2.11 & 62 & 1.94 & 75 & 3.07 & 104 \\
\hline Mean & 42 & & & & 466 & 2.44 & 63 & 1.57 & 52 & 1.30 & 37 \\
\hline
\end{tabular}

Pre-Tx $D x$, Diagnosis before lung transplantation; Day $R x$, postoperative day treatment with aerosolized cyclosporine was initiated; $F E F$, expiratory flow; $M$, male; $P F$, pulmonary fibrosis; $L$, left: $F$, female; $P H$, pulmonary hypertension; $R$, right; $C O P D$, chronic obstructive pulmonary disease; $C F$, cystic fibrosis.

*Percent of predicted.

In an attempt to address this issue we hypothesized that cyclosporine targeted directly into the allograft by aerosol inhalation would achieve a higher concentration than could be obtained by systemic delivery alone and would result in enhanced control of rejection. This hypothesis was confirmed in our experimental model of rat lung transplantation. ${ }^{7}$ Our previous studies that used gamma camera imaging of radiolabeled aerosolized cyclosporine indicated that high concentrations of cyclosporine can be delivered to the lung allograft in recipients with chronic allograft rejection ${ }^{8}$; however, the deposition pattern within the allograft is nonuniform. Subsequent studies showed that aerosolized cyclosporine can stabilize pulmonary function and reduce histologic inflammation in patients with obliterative bronchiolitis. ${ }^{9,10}$

In this study we evaluated the efficacy of aerosolized cyclosporine in the treatment of lung transplant recipients with acute cellular rejection that was refractory to conventional augmented immune suppression. Response to therapy was assessed by changes in the histologic grade of rejection and by changes in pulmonary function during aerosol treatment.

\section{Methods}

Patient demographics. The protocol for this phase II trial was approved by the Institutional Review Board of the University of Pittsburgh and informed consent was obtained from each patient. Between June 1993 and March 1996, 18 consecutive recipients with persistent acute rejection were given aerosolized cyclosporine. Patient characteristics are described in Table I. Entry into the study required refractory rejection as documented by transbronchial biopsy findings that persisted after multiple courses of pulse methylprednisolone and equine lymphocyte immune globulin (ATGAM) (mean $6.8 \pm$ 1.5 episodes). Acute rejection was considered significant if the grade was equal to or greater than grade $2 .{ }^{11}$ On average the patients had approximately 466 days of follow-up after transplantation before initiation of aerosolized cyclosporine treatment. Twenty-three recipients who had persistent refractory acute rejection before the development of aerosolized cyclosporine served as historical controls. Control patients were chosen to match for similar interval after transplantation, lung function, prevalence and treatment of rejection, type of lung transplant, age, and sex. Chronic rejection did not develop in any of these patients and they represent a different control population than the one used for a previous study. ${ }^{9}$ The incidence of acute rejection documented by transbronchial biopsy in this control population was on average $4.9 \pm 2.1$ episodes over an average of 390 days. 
Immunosuppressive regimen. Posttransplantation maintenance immunosuppressive regimens for all patients consisted of oral cyclosporine $(6 \mathrm{mg} / \mathrm{kg}$ per day, 7 recipients $)$ or tacrolimus $(0.30 \mathrm{mg} / \mathrm{kg}$ per day, 11 recipients), plus azathioprine $(2 \mathrm{mg} / \mathrm{kg}$ per day) and prednisone $(20 \mathrm{mg} /$ day). Oral doses of tacrolimus and cyclosporine were modified to maintain trough systemic levels at approximately 15 to $20 \mathrm{ng} / \mathrm{ml}$ (enzyme-linked immunosorbent assay) and 800 to $1000 \mathrm{ng} / \mathrm{ml}$ (polyclonal TDX assay), respectively, immediately after transplantation and throughout the course of this trial. The azathioprine dose was reduced when the leukocyte count fell to less than $5.0 \times 10^{6} / \mathrm{L}$. Enhanced immune suppression for treatment of acute rejection ( $\geq$ grade 2 ) consisted of either pulse methylprednisolone $(1.0 \mathrm{gm} /$ day for 3 days intravenously) or equine lymphocyte immune globulin (ATGAM) (20 $\mathrm{mg} / \mathrm{kg}$ per day for 14 days intravenously)

Histologic alssessment of rejection (before aerosol cyclosporine treatment). Fiberoptic bronchoscopy was routinely done approximately 2 weeks after transplantation and at 3-month intervals thereafter to detect asymptomatic rejection or infection. Transbronchial biopsy is recognized as an excellent means of detecting the vascular mononuclear infiltrate of acute rejection in the allograft and differentiating rejection from infection. ${ }^{12,13}$ Biopsy was done whenever changes in clinical or functional parameters such as oxyhemoglobin saturation, spirometry values ( $\geq 10 \%$ decline of forced vital capacity [FVC] or forced expiratory volume in 1 second $\left.\left[\mathrm{FEV}_{1}\right]\right)$, or chest radiographic findings occurred and at 4 to 6 weeks after pulse corticosteroid therapy and 6 to 8 weeks after equine lymphocyte immune globulin therapy to assess a treatment response. Acute cellular rejection was graded as A1 to A4 to indicate minimal, mild, moderate, or severe disease. ${ }^{11}$ Pathologists who interpreted biopsy samples were blinded to the immunosuppressive drug regimen.

Drug preparation, aerosol generation, and treatment protocol. Aerosolized cyclosporine was given with use of a commercially available jet nebulizer (Aero Tech II, CisUs, Bedford, Mass.) driven by compressed air (50 psi) at a flow rate of $10 \mathrm{~L} / \mathrm{min}^{14}$ Three hundred milligrams of cyclosporine (Sandoz, Basel, Switzerland) was dissolved in $4.8 \mathrm{ml}$ propylene glycol. Inhalation was done through a mouthpiece during spontaneous respiration. Treatments were given with use of a commercially available highefficiency particulate air filter to ensure absence of environmental contamination (AeroStar, BioSafety Systems, San Diego, Calif.).

Patients were premedicated by inhalation of $2 \%$ lidocaine $(5 \mathrm{ml})$ via a conventional nebulizer after which they received the aerosolized cyclosporine at a dosage of 300 mg per day. Daily peripheral blood samples were drawn for determination of trough cyclosporine concentrations and levels of creatinine and liver enzymes 24 hours after inhalation for the first 12 days of therapy. After completion of the initial 10-day schedule, treatments were given at home 3 days per week at $300 \mathrm{mg}$ per session.

Follow-up and outcome measures. Fiberoptic bronchoscopy with transbronchial biopsy was done under fluoroscopic guidance at approximately 4 weeks after the start of aerosolized cyclosporine therapy and then at 2- to 3-month intervals. A minimum of 10 tissue samples was obtained with alligator forceps at the same anatomic location (anterior, lateral, posterior basilar, or anteromedial, lateral, and posterior basilar segments of the right or left lower lobe, respectively) where acute rejection was detected before aerosolized cyclosporine therapy. Cyclosporine blood levels were measured and renal and liver function tests were done at 14-day intervals after discharge from the hospital. Pulmonary function tests and chest radiographs were done monthly and blood pressure and home spirometry values were measured by each patient and recorded daily.

Pharmacokinetics studies. To assess systemic absorption of aerosolized cyclosporine, blood cyclosporine levels were measured in four patients receiving oral tacrolimus as maintenance therapy. On the seventh day of aerosol treatment a trough cyclosporine level was obtained, followed by samples drawn on completion of the dose and at 20 minutes, 40 minutes, and 1, 2, 4, 8, and 24 hours after completion of the dose. Samples were analyzed by highperformance liquid chromatography. ${ }^{15}$

Statistical analysis. Random effects linear regression analysis was used to compare slopes of the FVC and FEV values after transplantation in the 18 patients who received aerosolized cyclosporine and in 23 control patients. In this regression model, pretreatment and posttreatment population slopes calculated for the aerosolized cyclosporine and control populations were analyzed for statistically significant slope differences $(p<0.05)$. For each pulmonary function test, we tested for a difference in the pretreatment period to determine whether to adjust for baseline differences between groups. Finding none, we fitted regression models to all subjects and tested for differences in population slopes in the posttreatment period. A Student's $t$ test was used to compare changes in the FVC, FEV 1 , and mean forced expiratory flow values immediately before and after approximately 200 days of aerosolized cyclosporine therapy. The rate of histologic rejection was compared before the start of aerosolized cyclosporine therapy and during maintenance aerosolized cyclosporine therapy with a Wilcoxon signed-rank test. Results are expressed as mean plus or minus the standard deviation.

\section{Results}

Changes in allograft histologic features during aerosolized cyclosporine therapy. All patients had grade 2 or higher rejection before treatment. Two of the patients (patients 10 and 14) were unable to tolerate the aerosol treatments because of intractable cough and airways irritation and had to be withdrawn from the trial. In $14(88 \%)$ of the remaining 16 subjects, the degree of histologic inflammation of acute rejection significantly diminished to minimal levels (A1) or disappeared (A0) with the institution of aerosolized cyclosporine therapy ( $\mathrm{Ta}$ ble II). One pediatric patient (patient 15 ) had only modest improvement as shown on the treatment biopsy results but was given continued therapy with subsequent improvement in pulmonary function 
Table II. Histologic changes in severity of rejection among 18 consecutive patients immediately before and during aerosolized cyclosporine therapy

\begin{tabular}{cccc}
\hline & \multicolumn{2}{c}{ Histologic grade } & Aerosol \\
\cline { 2 - 3 } Patient & Pretreatment & During treatment & $\begin{array}{c}\text { Areatment days* } \\
\text { tren }\end{array}$ \\
\hline 1 & A3 & A0 & 32 \\
2 & A2 & A0 & 29 \\
3 & A3 & A3 & 20 \\
4 & A2 & A0 & 37 \\
5 & A2 & A0 & 39 \\
6 & A2 & A0 & 70 \\
7 & A2 & A0 & 9 \\
8 & A3 & A1 & 12 \\
9 & A2 & A1 & 36 \\
$10 \dagger$ & A4 & A3 & 21 \\
11 & A3 & A0 & 38 \\
12 & A3 & A1 & 63 \\
13 & A3 & A1 & 38 \\
$14 \dagger$ & A3 & A3 & 37 \\
15 & A4 & A3 & 10 \\
16 & A2 & A0 & 78 \\
17 & A2 & A1 & 47 \\
18 & A2 & A1 & 57 \\
\hline
\end{tabular}

${ }^{*}$ Grades of acute rejection ( $\mathrm{A} 0$ through $\mathrm{A} 4$ ) are described in the text. Aerosol treatment days refers to duration of treatment before the follow-up biopsy was done.

$\nmid$ Patients 10 and 14 were unable to tolerate the aerosol treatments and were withdrawn from the trial.

testing results. Further biopsies have not been done. The other patient (patient 3) has not had any histologic improvement but has stabilized pulmonary function and so has been given continued therapy.

Sustained improvement in histologic rejection was seen in serial biopsy samples: the incidence of acute rejection (grade $\mathrm{A} 2$ or greater) decreased from $2.49 \pm 0.68$ per 100 patient days before therapy with aerosolized cyclosporine to $0.72 \pm 0.3$ per 100 patient days during therapy $(p<0.05)$. Patients have received aerosol treatments for an average of 187 days (range 105 to 246 days). Discontinuation of aerosolized cyclosporine was attempted in five patients but resulted in recurrence of histologic rejection in four of five recipients.

Pulmonary function before and during aerosolized cyclosporine therapy. To demonstrate the effect of aerosolized cyclosporine on pulmonary function, changes in $\mathrm{FVC}$ and $\mathrm{FEV}_{1}$ values were compared at similar intervals after lung transplantation in the 18 treated subjects and 23 matched control patients who had continued to receive conventional therapy (Fig. 1). Measures of FVC and $\mathrm{FEV}_{1}$ (change in percent predicted/100 days plus or minus the standard error) increased over time in the patients treated with aerosolized cyclosporine whereas pulmonary function in control patients declined despite repeated attempts at conventional rescue (FVC, aerosolized cyclosporine group, $4.6 \pm$ 2.9 vs control group, $-8.1 \pm 1.9, p=0.001 ; \mathrm{FEV}_{1}$, aerosolized cyclosporine group, $2.1 \pm 4.4$ vs control group, $-9.8 \pm 2.6, p=0.043$ ).

Pharmacokinetics of systemic cyclosporine after aerosolized inhalation. In four patients receiving oral tacrolimus, cyclosporine blood levels were measured during daily treatment with aerosolized cyclosporine at a $300 \mathrm{mg}$ dose. The average maximal blood level (by high-performance liquid chromatography) within 60 minutes of inhalation was $225 \pm$ $132 \mathrm{ng} / \mathrm{ml}$ (range 140 to $419 \mathrm{ng} / \mathrm{ml}$ ). Cyclosporine concentrations progressively decreased such that after 24 hours the mean trough cyclosporine level was $23 \pm 17 \mathrm{ng} / \mathrm{ml}$ (range $37 \mathrm{ng} / \mathrm{ml}$ to nondetectable). Renal function did not change significantly with the addition of aerosolized cyclosporine to the baseline immunosuppressive drug regimen.

\section{Discussion}

In this study we have shown that aerosolized cyclosporine was effective rescue therapy in 14 of 18 consecutive lung allograft recipients with unremitting histologic acute rejection. These patients had been given multiple courses of pulsed corticosteroids and antilymphocyte globulin but this therapy had failed to achieve a therapeutic response. Our previous trials had primarily concentrated on patients with refractory chronic rejection. ${ }^{9}{ }^{10}$ In these studies, treatment with aerosolized cyclosporine resulted in stabilization of pulmonary function as the histologic features of active inflammation improved. Application of this therapy in the treatment of persistent acute rejection is particularly important because of the significance of persistent acute rejection as a risk factor for the development of obliterative bronchiolitis. ${ }^{6}$

The results of this present study show that important definitive reductions in the histologic infiltrates of acute allograft rejection occurred on initiation of aerosolized cyclosporine therapy. Moreover, the changes observed in allograft histologic features during aerosolized cyclosporine therapy were associated with improvement in the spirometric indices of lung function. In contrast to findings in patients treated with aerosolized cyclosporine, a progressive decline in lung function was observed in a matched control population treated with conventional rescue 


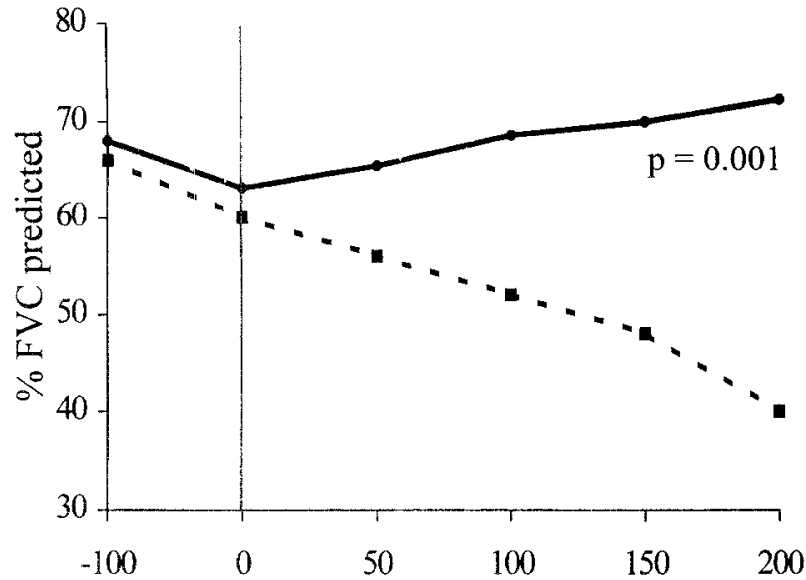

A

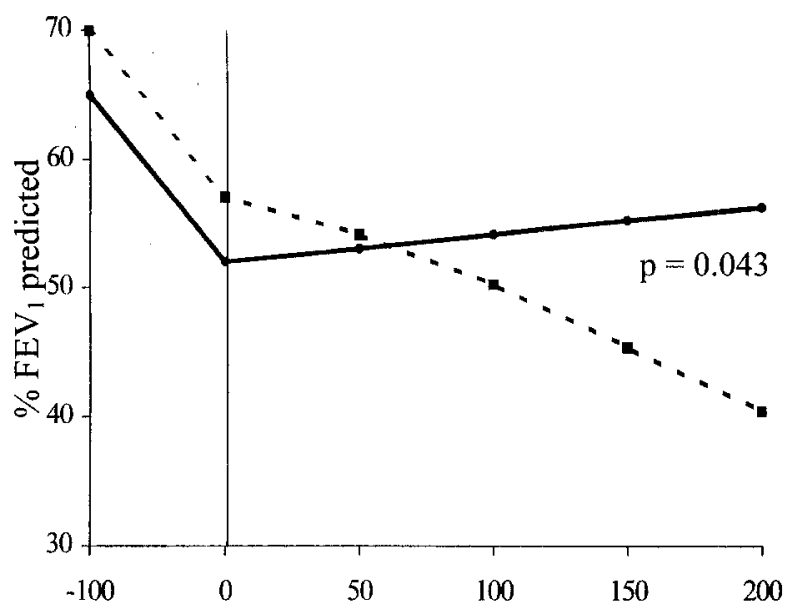

B

Aerosol Treatment Days

Fig. 1. Population regression lines showing changes in values of $F E V_{1}$ and $F V C$ (percent predicted) before and during 200 days of aerosolized cyclosporine therapy in 18 subjects and 22 control recipients, matched by prevalence of rejection, who received pulse methylprednisolone and cytolytic therapy. For the 18 subjects treated with aerosolized cyclosporine the percent predicted $F E V_{1}$ value actually improved whereas there was no comparable interruption in the decline in values of either FVC ( $p=0.001)$ or FEV $(p=0.043)$ in the control population.

immunosuppression. These results indicate that inhaled cyclosporine is more effective than pulsed methylprednisolone and antilymphocyte globulin in reversing persistent acute histologic rejection. The effects of aerosol treatment appear to be sustained because repeated transbronchial biopsy samples obtained at defined intervals have shown consistent reductions in the grades of acute rejection.

The most frequent adverse effect related to aerosolized cyclosporine treatment consisted of cough and transient dyspnea. Although most subjects had these effects during inhalation, only two patients required premature discontinuation of the treatment. These two patients do, however, demonstrate that airways irritation may be severe enough to limit the use of aerosolized cyclosporine in some recipients.

The absence of significant systemic absorption after inhalation of cyclosporine would probably account for the lack of renal and hepatic toxicity in our study population. We have also shown that whole blood levels of cyclosporine are minimal after inhalation of a $300 \mathrm{mg}$ dose of cyclosporine. These results support our hypothesis that the inhaled route of administration for cyclosporine $\mathrm{A}$ is safe.

The efficacy of aerosolized cyclosporine therapy has encouraged us to embark on a prospective randomized trial of this agent compared with standard rescue treatments. Our protocol will compare a limited course of aerosolized cyclosporine versus methylprednisolone when the second episode of acute rejection is documented. All patients will receive methylprednisolone for a first rejection event. All patients will undergo transbronchial biopsy with histologic and cytokine analysis of bronchoalveolar lavage fluid and peripheral blood. Subjects who receive aerosolized cyclosporine will also undergo testing to measure the regional deposited amounts of inhaled cyclosporine within the transplanted lung or lungs and to correlate these findings with changes in histologic rejection and alterations of cytokine gene expression. The protocol calls for a limited ( 8 to 12 weeks) treatment course with aerosolized cyclosporine. Patients in both groups who have treatment failure will be given cytolytic therapy.

Improvements in the histologic grade of rejection after aerosolized cyclosporine therapy appear to correlate with downregulation of proinflammatory cytokine gene expression for interleukin-6 and interferon- $\gamma$ in cells of bronchoalveolar lavage fluid. ${ }^{10}$ This immunomodulation may also account for the stabilization of pulmonary function measures in the two patients in whom histologic resolution was not 
seen. The proposed randomized trial should yield better information to elucidate the mechanism of action of this agent.

It is recognized that there are regional differences in cytokine expression, and, similarly, varying degrees of severity of rejection within the lung allograft have been documented. In addition, deposition of aerosolized drug within the lung is clearly not uniform. ${ }^{8}$ Under the proposed protocol, biopsy specimens will be obtained from various sites according to the deposition studies. This information will be necessary to determine the optimal candidates for acrosolized cyclosporine therapy and to maximize efficacy of this therapy.

This study has demonstrated that aerosolized cyclosporine was more effective than conventional treatment in suppressing the inflammatory response in the allograft of lung transplant recipients with refractory acute rejection. We speculate that aerosolized cyclosporine also has the potential to allow reduction in oral doses of immunosuppressive agents thereby further reducing the risk of infection and systemic toxicity. Ultimately, improved control of acute rejection with aerosolized cyclosporine may prevent the subsequent phase of chronic allograft rejection, which is characterized by irreversible lung injury as a result of fibrosis in the transplanted lung.

\section{REFERENCES}

1. Trulock EP. Management of lung transplant rejection. Chest 1993;103:1566-76.

2. Griffith BP, Hardesty RL, Armitage JM, et al. Acute rejection of lung allografts with various immunosuppressive protocols. Ann Thorac Surg 1992;54:846-51.

3. Paradis I, Yousem S, Griffith BP. Airway obstruction and bronchiolitis obliterans after lung transplantation. Clin Chest Med 1993;14:751-63.

4. Kesten S, Maidenberg A, Winton T, Maurer J. Treatment of presumed and proven acute rejection following six months of lung transplant survival. Am J Respir Crit Care Med 1995; 152:1321-4.

5. Keenan RJ, Konishi H, Paradis IL, et al. A clinical trial of tacrolimus versus cyclosporine in lung transplantation. Ann Thorac Surg 1995;60:580-5.

6. Bando K, Paradis IL, Konishi $\mathrm{H}$, et al. Obliterative bronchiolitis after lung and heart-lung transplantation: an analysis of risk factors and management. J Thorac Cardiovasc Surg 1995;110:4-14.

7. Keenan RJ, Duncan AJ, Yousem SA, et al. Improved immunosuppression with aerosolized cyclosporine in experimental pulmonary transplantation. Transplantation 1992;53: 20-5.

8. O'Riordan TG, Iacono AT, Keenan RJ, et al. Delivery and distribution of aerosolized cyclosporine in lung allograft recipients. Am J Respir Crit Care Med 1995;151:516-21.

9. Iacono AT, Keenan RJ, Duncan SR, et al. Aerosolized cyclosporine in lung recipients with refractory chronic rejection. Am J Respir Crit Care Med 1996;153:1451-5.

10. Keenan RJ, Zeevi A, Iacono AT, et al. Efficacy of inhaled cyclosporine in lung transplant recipients with refractory rejection: correlation of intragraft cytokine gene expression with pulmonary function and histologic characteristics. Surgery 1995;118:385-91.

11. Yousem SA, Berry GJ, Brunt EM, et al. A working formulation for the standardization of nomenclature in the diagnosis of heart and lung rejection: lung rejection study group. J Heart Lung Transplant 1990;12:713-6.

12. Trulock EP, Ehinger NA, Brunt EM, Pasque MK, Kaiser LR, Cooper JD. The role of transbronchial biopsy in the treat ment of lung transplant recipients. Chest 1992;102:1049-54.

13. Tazelaar HD, Nilsson FN, Rinaldi M. The sensitivity of transbronchial biopsy for the diagnosis of acute lung rejection. J Thorac Cardiovasc Surg 1993;105:674-8.

14. O'Riordan TG, Duncan SR, Burckart GJ, Griffith BP, Smaldone GC. Production of an aerosol of cyclosporine: a prelude to clinical studies. J Aerosol Med 1992;5:171-7.

15. Burckart GJ, Venkataramanan R, Ptachcinski RJ, et al. Cyclosporine absorption following orthotopic liver transplantation. J Clin Pharmacol 1986;26:647-51.

\section{Discussion}

Dr. Sara J. Shumway (Minneapolis, Minn.). We know that the efficacy of aerosol therapy is influenced by a number of variables. These include the type of nebulizer device used to deliver the aerosol, the physical properties of the drug, its concentration in the aerosol and particle size, its carrier gas, and the somewhat nebulous catch-all known as patient factors. From previous studies, primarily by the Pittsburgh group, we know that pulmonary deposition of cyclosporine has been demonstrated after aerosolized cyclosporine administration. It appears to accumulate in the trachea, bronchi, and regional lymph nodes as well.

This study reports findings in a relatively small group of patients without a real control group. If the authors were to initiate randomized controlled trials with aerosolized cyclosporine, what would they test it against? Methotrexate has been used with some benefit in certain patients. What about photopheresis?

The maintenance protocol the authors used may require modification. Perhaps these patients need to be given more oral cyclosporine rather than receiving it in the aerosolized form. Three hundred milligrams is quite a lot of cyclosporine. What sort of cyclosporine levels occur when the patients are receiving both oral and aerosolized cyclosporine? The cost of the added aerosolized cyclosporine is yet another consideration.

Finally, I believe the real target population for aerosolized cyclosporine therapy may be patients who undergo bilateral single-lung or heart-lung transplantation because of cystic fibrosis. They are usually used to receiving antibiotics in this fashion before transplantation and have trouble with adequate absorption after oral ingestion of cyclosporine. This paper presents an excellent pilot study that demonstrates this treatment option for persistent acute lung allograft rejection. 
Dr. Keenan. We did not mention in the presentation here a proposal that we have been debating on whether to randomize these patients much earlier in the course of the transplantation treatment. After the first episode of rejection treated by standard therapy, should we then go on to using aerosolized cyclosporine in place of yet another dose of methylprednisolone or, potentially, cytolytic therapy? We are looking at this issue of where the potential role of aerosolized cyclosporine lies.

We also have been using alternative therapies to deal with persistent or refractory rejection. We have been using methotrexate in a number of patients. Photopheresis is available, although it too is expensive. The kits are available, but the time it takes for delivery and so on leads to a considerable expense. Also, although there have been a few patient series reported and anecdotal reports of this therapy in lung transplant recipients, it, too, is in a sense an experimental therapy, and therefore to use one experimental therapy and judge it against another experimental therapy I think would be stretching the issue. That is why we have chosen to go this route, which is the gold standard, if you will, of methylprednisolone or antithymocyte globulin versus aerosolized cyclosporine.

In terms of the inhalation of these agents, we too were concerned, particularly early on, that the single-lung recipients with the intact native lung and the intact neuroconduits would be less able to tolerate the solution, and, in fact, this is what we found in several patients who had chronic rejection. In the patients with acute rejection, that has not been as consistent a problem, and of the two patients in whom the therapy had to be discontinued, one was a double-lung recipient and one was a single-lung recipient. Thus we have not yet found the need to discriminate between single- and double-lung recipients in terms of delivery of the agent.

We have done a considerable amount of work with a colleague, Jerry Smaldone, in Stony Brook to examine the issue of particle size and distribution. His work with radio-tagged albumin as a correlate to the inhaled cyclosporine shows that the vast majority of this drug is deposited into the parenchyma with a mean aerodynamic diameter of approximately $1.2 \mu \mathrm{m}$, and this is fairly consistent. The beauty of the particular nebulizer that we are using is that one can show that the particle size distribution does not change from the beginning of the nebulized treatment to the end of the treatment; thus we are getting a consistent pattern of deposition.

I cannot tell you exactly the levels of cyclosporine in all of these patients. What I can tell you is that we use a TDX method for our cyclosporine surveillance and maintain the level between 800 and $1000 \mathrm{ng} / \mathrm{ml}$, and we did not have to adjust the systemic cyclosporine level in any of the patients to account for the increased absorption from the aerosol.

\section{Availability of Journal back issues}

As a service to our subscribers, copies of back issues of The Journal of Thoracic and Cardiovascular Surgery for the preceding 5 years are maintained and are available for purchase from Mosby at a cost of $\$ 15.00$ per issue until inventory is depleted. The following quantity discounts are available: $25 \%$ off on quantities of 12 to 23, and one third off on quantities of 24 or more. Please write to Mosby-Year Book, Inc., Subscription Services, 11830 Westline Industrial Drive, St. Louis MO 63146-3318, or call 800-453-4351 or 314-453-4351 for information on availability of particular issues. If unavailable from the publisher, photocopies of complete issues may be purchased from UMI, 300 N. Zeeb Rd., Ann Arbor, MI 48106, 313-761-4700. 